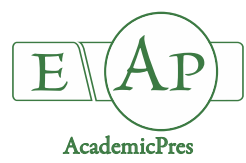

\title{
High Frequency $2 n$ Pollen Formation in Black Poplar (Populus nigra L.) Induced by Colchicine
}

\author{
Wenting XU ${ }^{1,2}$, Yan $Z H A N G{ }^{1}$, Liqin $\mathrm{GUO}^{1,3}$, Jian $\mathrm{ZHAO}^{1}$, \\ Zunzheng $\mathrm{WEI}^{4 *}$, Jinfeng $\mathrm{ZHANG}^{1 *}$ \\ ${ }^{1}$ Beijing Forestry University, College of Biological Sciences and Technology, Beijing Advanced Innovation Center for Tree Breeding by Molecular \\ Design, National Engineering Laboratory for Tree Breeding, Key Laboratory of Genetics and Breeding in Forest Trees and Ornamental Plants of \\ Ministry of Education, Key Laboratory of Forest Trees and Ornamental Plants, Biological Engineering of State Forestry Administration, \\ Beijing, 100083, P. R. China; zjf@bjfu.edu.cn (*corresponding author) \\ ${ }^{2} Z$ hejiang A\& F University, School of Forestry and Biotechnology, State Key Laboratory of Subtropical Silviculture, \\ Hangzhou, 311300, P. R. China \\ ${ }^{3}$ Beijing Academy of Forestry and Pomology Sciences, Beijing, 100093, P. R. China \\ ${ }^{4}$ Beijing Academy of Agriculture and Forestry Sciences, Key Laboratory of Biology and Genetic Improvement of Horticultural Crops (North \\ China), Ministry of Agriculture, Key Laboratory of Urban Agriculture (North), Ministry of Agriculture, Beijing Vegetable Research Center, \\ Beijing, 100091, P.R. China; weizunzheng@163.com (*corresponding author)
}

\begin{abstract}
Populus nigra L. is one of the most important male genetic donors in populus genetic improvement and tree breeding over the world. Many excellent Populus nigra clones are identified as triploids potentially obtaining by hybridization of $2 n$ pollen and normal oogamete. This study revealed the cytological mechanism of $2 n$ pollen formation in Populus nigra L. for the first time and concluded the best treated combination of colchicine treatment, which obtained $2 n$ pollen with the highest rate reaching at $87.11 \%$ (even $100 \% 2 n$ pollen in some floral buds) which made the polyploid hybridization utilizing $2 n$ pollen in section Aigeiros possible.
\end{abstract}

Keywords: $2 n$ pollen; colchicine; Populus nigra L.

\section{Introduction}

Polyploidy is an intriguing phenomenon that is regarded as an important mechanism of speciation and adaptation in plants (Otto and Whitton, 2000). A polyploid is simply an organism that contains more than two complete sets of chromosomes. It commonly arises from mitotic or meiotic misdivisions by nondisjunction of chromosomes. Polyploids differ from their diploid progenitors in morphological, ecological, physiological and cytological characteristics. Its features, such as broader and thicker leaves, larger flowers, longer internodes, fewer stems per plant, fewer inflorescences, higher vegetative yields, more compact plants, greater tolerance to environmental stress, higher (additive) resistance to several diseases, increased seed weight but fewer seeds or higher chlorophyll production
(Dewitte et al., 2012), endow them with important breeding value on a more practical level.

Gametes with somatic chromosome numbers also referred to as $2 n$ gametes or (numerically) unreduced gametes are considered the driving force behind the formation of polyploids in nature (Bretagnolle and Thompson, 1995; Otto and Whitton, 2000). It originates from meiotic aberrations. $2 n$ gametes are an effective and efficient way to transmit genetic diversity (allelic variation) to cultivated forms, including both valuable qualitative and quantitative traits. More recently, plant breeders have become interested in the practical use of $2 n$ gametes in breeding program due to the new tools available for $2 n$ gamete manipulation and insights into the genetic background of their formation (Dewitte et al., 2012).

Formation of $2 n$ gametes can be described as two main categories: first division restitution (FDR) and second division restitution (SDR) (Ramanna, 1983; Jongedijk et al., 1991; Bretagnolle and Thompson, 1995; Ramanna et al., 
940

2003; Zhou et al., 2008). In FDR, homologous chromosomes separation does not occur at meiosis I while sister chromatids of each chromosome normally moved to opposite poles at meiosis II. After cytoplasm division, FDR gametes retain all parental chromosomes without consideration of crossover segments. In SDR, chromosomes have normal behavior at meiosis I, but chromatids do not migrate to the two poles after centromeres divided at meiosis II. Therefore, SDR gametes contain two copies of genetic information as in normal gametes. Furthermore, aberrations during spindle formation such as parallel spindle, tripolar spindle, fused spindle might result in triads, dyads, or monads (Bretagnolle, 1995; Taschetto and Pagliarini, 2003; Dewitte et al., 2010;) which would following produce FDR or SDR gametes based on a certain proportion. Besides, indeterminate meiosis restitution (IMR) (Lim et al., 2001) and post meiosis restitution (PMR) (Bastiaanssen et al., 1998) were reported in some species, which can produce $2 n$ gametes equivalent to FDR or SDR gametes.

However, rate of natural $2 n$ pollen production is too low to satisfy cross breeding in popular (Zhu et al., 1998; Kang and Zhu, 1997; Li, 2001), so artificial induction must be implemented to improve yield of $2 n$ pollen with the purpose of polyploidy acquisition. $2 n$ pollen can be induced by several physical factors such as temperature, radial ray, ultrasonic, and centrifugal force among which the high temperature is the most applied one (Mashkina et al., 1989; Li et al., 2000; Kang et al., 2000). While colchicine as representative of chemical factors is more generally used to induce $2 n$ pollen by blocking up the formation of spindle (Salmon et al., 1984).

Populus tomentosa $\times P$. bolleana was treated with colchicine and gained $2 n$ pollen reaching up to $88 \%$ (Kang et al., 1999). In P. psedo-simonii Kitag, rates of unreduced pollen induced by colchicine and high temperature were 60.69\% and over 59\%, respectively. In $P$. simonii $\times P$. nigra 'Tongliao', production of unreduced pollen was reached at $30.75 \%$ induced by colchicine and $50.28 \%$ induced by high temperature. Populus $\times$ popularis were induced by colchicine and the rate of $2 n$ pollen was $62.10 \%$ (Xi et al., 2011). In sect. Aigeiros, production of unreduced gametes in $P$. deltoides Marsh. cv. 'Shanghaiguanensis' and $P . \times$ euramericana were $87.40 \%$ and $95.44 \%$ improved by artificial induction, respectively. While in $P$. nigra var. Italica (Moench.) Koehne, an original species in sect. Aigeiros, production of $2 n$ pollen was lower than the two hybrids mentioned above (55.67\%) (Zhang, 2006). Hybrids, some of which can produce natural $2 n$ pollen, were chosen in many studies and had favorable performance in artificial induction probably because of their unstable genomes that may be doubled more easily (Kang, 2002; Zhang and Kang et al., 2010; Tian et al., 2015). Research on original species in Populus is still at an early stage, and the cytological mechanisms of unreduced gametes formation are not clear.

Populus nigra L. is one of the original species in sect. Aigeiros which is widespread with the altitude range of 400$600 \mathrm{~m}$ around West and Middle Asia. It plays an important role in populus genetic improvement and tree breeding as the male genetic donor of the major popular cultivars over the world. Many excellent clones in section Aigeiros such as P. $\times$ euramericana 'Zhonglin- 46 ', $P . \times$ langfangensis- $3, P . \times$ canadensis 'I-214' which have widely generalized in China have been all identified as triploids potentially obtained by hybridization of $2 n$ pollen and normal oogamete (Zhang et al., 2004). To investigate the best condition of colchicine induction and cytological mechanisms of unreduced gametes formation, three male genome types of Populus nigra L. ('TL', 'XJ1' and 'XJ2') from two localities were used in this study.

\section{Materials and Methods}

\section{Plantmaterial}

Flower branches of 'TL', 'XJ1' and 'XJ2' were collected from Tongliao in the Nei Mongol Autonomous Region ('TL') and Altay Prefecture in the Xinjiang Uygur Autonomous Region ('XJ1' and 'XJ2'), receptively. All branches with robust floral buds were trimmed into 150$200 \mathrm{~cm}$, sealed with plastic film and transported to Beijing Forestry University. Water culture was performed in greenhouse and the water was changed every 3 days.

\section{(PMCs)}

Observation of floral buds, catkins and pollen mother cells

Floral buds and catkins were photographed by digital camera Sony DSC-W 70 while florets and anthers were observed by stereomicroscope Olympus SZX12 and photographed by digital camera Olympus C5060 Wide Zoom.

Treated buds were collected and fixed with Carnoy's Fluid (70\% ethanol/acetic acid, 3:1) at 0, 3, 6, 9 and $12 \mathrm{~h}$ after colchicine injection and each sample contained 3-5 buds. After fixing overnight buds were transferred into $70 \%$ ethanol for storage at $4^{\circ} \mathrm{C}$.

Temporary tablet productions of PMCs dyed with acetocarmine were observed by optical microscope Olympus BX-51 and photographed by photographic system Olympus DP 70. Contrast and brightness of all photographs were processed by Adobe Photoshop CS4.

\section{Colchicine injection}

Process of PMCs development is related to the environmental temperature ( $\mathrm{Xu}$ et al., 2011), so that temperature in greenhouse was recorded when the floral branches were started to water cultivate. Anthers at the middle part of the fastest developed floral bud were collected to assess the process of PMCs development every 3 $h$ at daylight when the floral branches had cultivated for 4 days. According to Zhang's research, colchicine injection would be performed when PMCs started to enter pachytene (Fig. 1D) (Zhang, 2006). Experiment must be completed mainly at daylight so that effect of colchicine could cover meiosis process as long as possible (Xu et al., 2011). If PMCs entered pachytene stage at afternoon, branches would be moved to cold room where the temperature remain at $5{ }^{\circ} \mathrm{C}$ for the development of PMCs would be suspended (Kang et al., 1999). In this case, injection would be performed the next day. 



Fig. 1. Appearance of floral bud, floret and anther when the PMCs started to enter pachytene A) floral buds; B) floret; C) anter; D) PMC in pachytene

In order to avoid the asynchrony of PMCs development, peaks of all floral buds for injection were removed previously (Xu et al., 2011). The rest parts were injected with disposable syringes and liquid injected into the floral buds was about $50 \mu \mathrm{l} /$ time/floral bud to ensure the floral bud infiltrated with solution. Experiments which were implemented in three different genotypes 'XJ1', 'XJ2', and 'TL' respectively were performed with three factors: concentration of colchicine, interval time, and times of injection following by Orthogonal Design $\mathrm{L}_{9}\left(3^{4}\right)$ (Table 1). The control group followed all the procedures mentioned above and replaced colchicine with distilled water.

\section{Pollen collection and statistics}

Pollen were collected into vials with silica gel desiccant respectively according to the treatment combination and stored at $4{ }^{\circ} \mathrm{C}$ before used. Statistics of $2 n$ pollen rate was performed under optical microscope Olympus BX-51 that every treatment combination must include at least 15 floral buds and 300 pollen.

\section{Results}

\section{Damage of floral buds}

Colchicine injection brought the floral buds both chemical and physical damages and made the subsequent development suffocated (Fig. 2), while buds injected with distilled water (Fig. 2A, D, G) were only suffered physical damage which blocked the rachis to elongate. However, pollen production of these buds was normal compared with floral buds without any treatment.

\section{Pollen observation and statistics of $2 \mathrm{n}$ pollen rate}

PMCs entered pachytene stage at 15:00 in the $6^{\text {th }}$ day after branches started to water cultivate. The branches were moved to cold room subsequently and the injection was performed the next day. Temperature in greenhouse at night was below $15{ }^{\circ} \mathrm{C}$ before PMCs entered pachytene stage (Table 2).

Table 1. Orthogonal design $\mathrm{L}_{9}\left(3^{4}\right)$ of colchicine injection

\begin{tabular}{cccc}
\hline Levels & \multicolumn{2}{c}{ Factors } \\
\cline { 2 - 4 } & & Concentration of colchicine $(\%)$ & 5 \\
2 & 0.4 & 4 & 4 \\
3 & 0.6 & 3 & 5 \\
\hline
\end{tabular}

Table 2. Temperature in greenhouse after the floral branches started to water cultivate $\left({ }^{\circ} \mathrm{C}\right)$

\begin{tabular}{cccccc}
\hline Day & & & Time & \\
\cline { 2 - 6 } & $9: 00$ & $12: 00$ & $15: 00$ & $18: 00$ & $21: 00$ \\
\hline 1 & 11 & & 18 & 12 & 12 \\
2 & 14 & & & 18 \\
3 & 13 & 15.5 & 15 \\
4 & 13 & 14 & & 14 \\
5 & 14 & 18 & 11 & 16 \\
6 & 9 & & 15 & 17 \\
7 & 10 & 17 & 10 & 13 & 13 \\
8 & 12 & 25 & 23 & & 22 \\
9 & 10 & & 26 & & 13 \\
10 & 13 & & & & \\
\hline
\end{tabular}




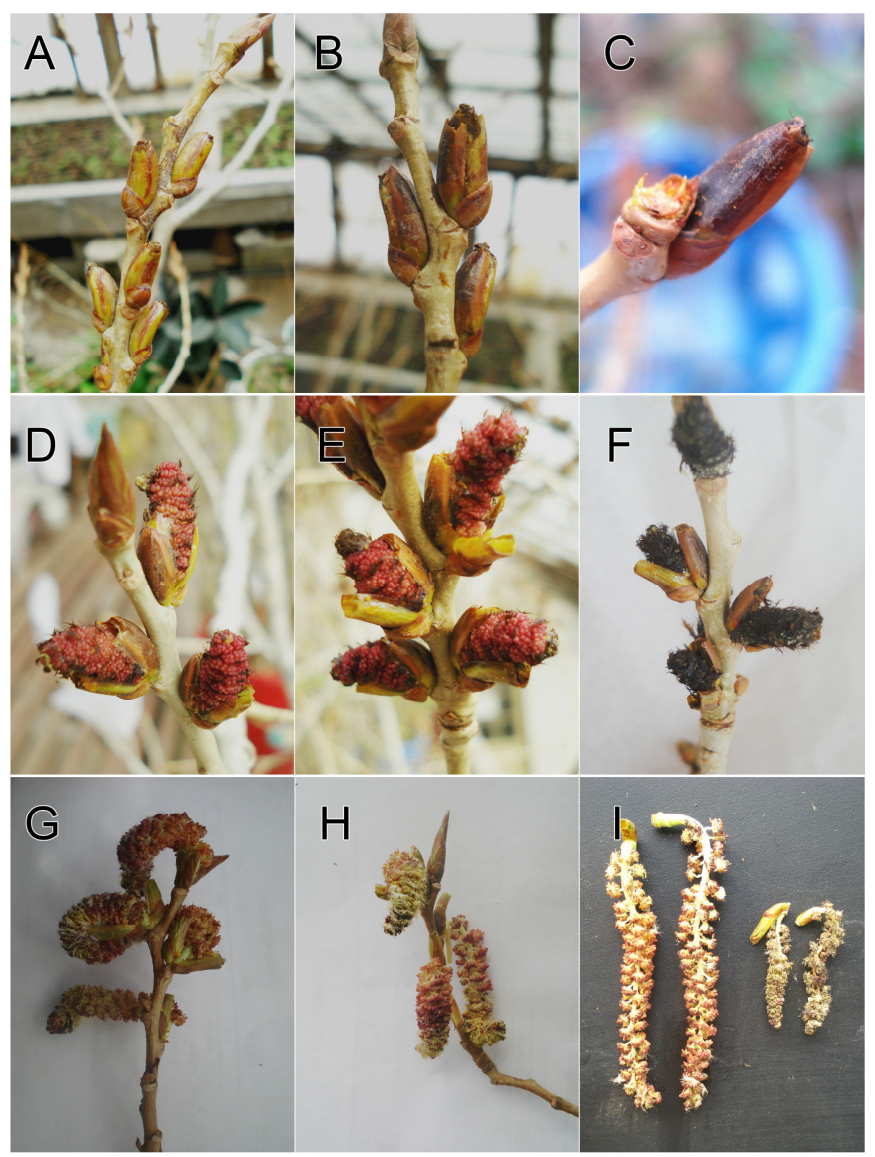

Fig. 2. Damage of floral buds in different development stages A) buds $24 \mathrm{~h}$ after distilled water injection; B, C) buds $24 \mathrm{~h}$ after colchicine injection; D) buds 10 days after distilled water injection; E, F) buds 10 days after colchicine injection; G) buds 15 days after distilled water injection; $\mathrm{H}$ ) buds 15 days after colchicine injection; I) comparison of normal developed buds (left) and buds with colchicine injection

The relationship between development process of PMCs and environmental temperature was consistent with our previous conclusion (Xu et al., 2011). The result that leptotene appeared at Day 6th which was consistent with the conclusion we reached previously reconfirmed the relationship between temperature and development process of PMCs.
Anthers started dehisce and pollen was collected in the 12 th day after injection. The diameter of $2 n$ pollen showed mainly about twice the normal one after colchicine induction (Fig. 3A). Few of $2 n$ pollen were observed in control group (Fig. 3B) probably due to the conservative meiosis of Populus nigra $\mathrm{L}$. as one of the original species in sect. Aigeiros (Xu et al., 2011).

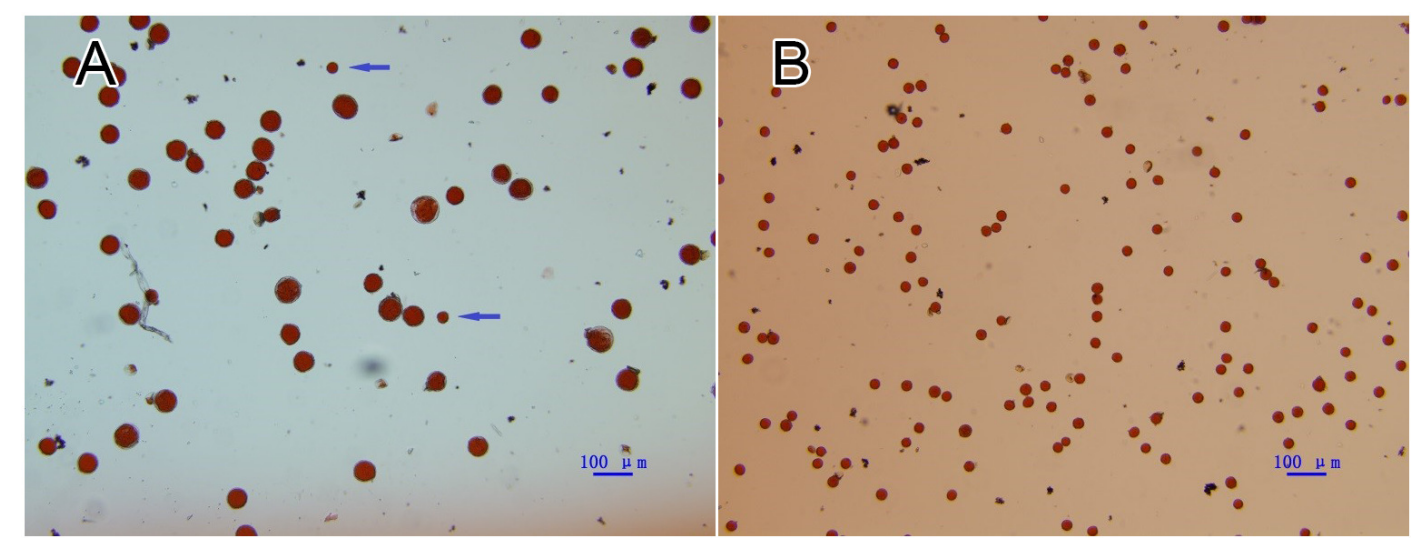

Fig. 3. Comparison of $2 n$ pollen and $I n$ pollen A) $2 n$ pollen after colchicine induction (arrows showed the $1 n$ pollen); B) $1 n$ pollen in control group (no $2 n$ pollen found) 
Results of orthogonal test in three genotypes and range analysis of three factors involved in the test were illustrated in Tables 3-4. The highest rate of $2 n$ pollen was $87.11 \%$ in genotype 'XJ1' (even in some floral buds we observed 100\% $2 n$ pollen) injected with $0.8 \%$ colchicine solution 4 times every 3 hours. This result was $31.44 \%$ increase compared with Zhang's work in sect. Aigeiros (Zhang, 2006) and in close proximity to the highest $2 n$ pollen yield of artificial induction in Populus hybrids (Kang et al., 1999; Zhang, 2006), indicating that original species in Populus can obtain $2 n$ pollen for hybridization via colchicine induction.

According to the range analysis, the best combination of colchicine injection was obtained in every genotype even though this combination did not exist in orthogonal test. Various responses of different genotypes to colchicine injection revealed that genotype was an important factor influenced the production of $2 n$ pollen in colchicine induction. This result indicated that selection of genotype before artificial induction and hybridization was necessary and pivotal for polyploid breeding in Populus.

In genotype 'TL', the highest $2 n$ pollen rate was reached $56.83 \%$ at test No. 5 , the best combination was $0.6 \%$ of colchicine injected 3 times every 3 hours. The most influential factor in this genotype was concentration of colchicine and the second one was times of injection, while interval time of colchicine injection had little effect. Average rate of $2 n$ pollen was peaked to $47.33 \%$ when colchicine concentration was $0.6 \%$ and then obviously decreased when colchicine concentration reached up to $0.8 \%$, suggesting that genotype 'TL' was susceptive to colchicine and high concentration of colchicine restrained the production of $2 n$ pollen. Though interval time had little effect on $2 n$ pollen production, we still observed a decreasing tendency in average rate of $2 n$ pollen with the increase of interval time. $2 n$ production was maximized when the injection performed 3 times. In genotype 'XJ1', the highest $2 n$ pollen rate was reached $87.11 \%$ at test No. 9 , the best combination was $0.8 \%$ of colchicine injected 5 times every 3 hours. The most influential factor in this genotype was concentration of colchicine and the second one was interval time, while times of injection had the least effect on $2 n$ pollen production. $2 n$ pollen production was unsatisfactory when colchicine concentration was $0.4 \%$ or $0.6 \%$, while $0.8 \%$ colchicine solution made markedly better performance on producing $2 n$ pollen in this genotype. $2 n$ pollen production was observably higher when floral buds were injected every 3 hours compared with the other two levels. Addition of injection times increased the production of $2 n$ pollen and 5 times injection got the best result. There was no $2 n$ pollen at test No. 6 probably because hysteresis or premature of PMCs development process in this test made the colchicine treatment incapable of covering their meiosis process.

In genotype 'XJ2', the highest $2 n$ pollen rate was reached $84.72 \%$ at test No. 8 , the best combination was $0.8 \%$ of colchicine injected four times every five hours. The most influential factor in this genotype was interval time and the second one was concentration of colchicine, while times of injection had little effect on production of $2 n$ pollen. $0.8 \%$ colchicine solution was more efficient compared with the other levels, while deleterious effect of high concentration on the floral buds was also strong. Pollen production was reduced significantly. At test No. 9, all floral buds were blackened and sere and finally no pollen was obtained which probably because of exorbitant concentration of colchicine and frequent injection. Influence of interval time over ' $\mathrm{XJ2}$ ' was most significant which was different from the other two genotypes.

This genotype was sensitive to injection operation and longer interval time was beneficial to high production of $2 n$ pollen. Times of injection had little effect on the production of $2 n$ pollen, and 4 times injection operation achieved the highest $2 n$ pollen production.

Table 3. Results of $2 n$ pollen induction by colchicine

\begin{tabular}{|c|c|c|c|c|c|c|}
\hline \multirow{2}{*}{$\begin{array}{l}\text { Test } \\
\text { No. }\end{array}$} & \multirow{2}{*}{$\begin{array}{c}\text { Concentration of } \\
\text { colchicine }(\%)\end{array}$} & \multirow{2}{*}{$\begin{array}{c}\text { Interval time } \\
\text { (h) }\end{array}$} & \multirow{2}{*}{$\begin{array}{l}\text { Times of } \\
\text { injection }\end{array}$} & \multicolumn{3}{|c|}{ Rate of $2 n$ pollen (\%) } \\
\hline & & & & $\mathrm{TL}$ & $\mathrm{XJ1}$ & $\mathrm{XJ} 2$ \\
\hline 1 & 0.4 & 5 & 3 & $56.33 \pm 0.06$ & $13.78 \pm 0.05$ & $25.60 \pm 0.08$ \\
\hline 2 & 0.4 & 4 & 4 & $28.30 \pm 0.04$ & $6.84 \pm 0.02$ & $63.18 \pm 0.07$ \\
\hline 3 & 0.4 & 3 & 5 & $55.29 \pm 0.07$ & $46.90 \pm 0.16$ & $19.75 \pm 0.03$ \\
\hline 4 & 0.6 & 5 & 4 & $36.91 \pm 0.03$ & $7.18 \pm 0.03$ & $69.42 \pm 0.10$ \\
\hline 5 & 0.6 & 4 & 5 & $56.83 \pm 0.10$ & $10.41 \pm 0.02$ & $13.30 \pm 0.03$ \\
\hline 6 & 0.6 & 3 & 3 & $48.24 \pm 0.05$ & 0.00 & $4.35 \pm 0.02$ \\
\hline 7 & 0.8 & 5 & 5 & $14.97 \pm 0.06$ & $56.36 \pm 0.14$ & $80.42 \pm 0.08$ \\
\hline 8 & 0.8 & 4 & 3 & $30.07 \pm 0.13$ & $31.62 \pm 0.07$ & $84.72 \pm 0.04$ \\
\hline 9 & 0.8 & 3 & 4 & $18.73 \pm 0.04$ & $87.11 \pm 0.06$ & 0.00 \\
\hline
\end{tabular}

Table 4. Range analysis of $2 n$ pollen induction by colchicine

\begin{tabular}{|c|c|c|c|c|c|c|c|c|c|c|c|}
\hline \multicolumn{4}{|c|}{ Concentration of colchicine } & \multicolumn{4}{|c|}{ Interval time } & \multicolumn{4}{|c|}{ Times of injection } \\
\hline \multirow{2}{*}{ Level (\%) } & \multicolumn{3}{|c|}{ Average rate (\%) } & \multirow{2}{*}{ Level (h) } & \multicolumn{3}{|c|}{ Average rate (\%) } & \multirow{2}{*}{ Level (h) } & \multicolumn{3}{|c|}{ Average rate $(\%)$} \\
\hline & TL & $\mathrm{XJ} 1$ & $\mathrm{XJ} 2$ & & $\mathrm{TL}$ & $\mathrm{XJ} 1$ & $\mathrm{XJ} 2$ & & $\mathrm{TL}$ & $\mathrm{XJ} 1$ & $\mathrm{XJ} 2$ \\
\hline 0.4 & 46.64 & 22.51 & 36.18 & 5 & 36.07 & 25.77 & 58.48 & 3 & 44.88 & 15.13 & 38.22 \\
\hline 0.6 & 47.33 & 5.86 & 29.02 & 4 & 38.40 & 16.29 & 53.73 & 4 & 27.98 & 33.71 & 44.20 \\
\hline 0.8 & 21.26 & 58.36 & 55.04 & 3 & 40.75 & 44.67 & 8.03 & 5 & 42.36 & 37.89 & 37.82 \\
\hline $\mathrm{R}$ & 26.07 & 52.50 & 26.02 & $\mathrm{R}$ & 4.68 & 28.38 & 50.45 & $\mathrm{R}$ & 16.90 & 22.76 & 6.38 \\
\hline
\end{tabular}


Every genotype had a best treatment combination respectively, while after an overall consideration of the statistics data, orthogonal design analysis, experimental operation and subsequent development of floral buds after injection, the ultimate best treatment combination was obtained as using $0.6 \%$ colchicine to inject peak-removed floral buds in pachytene 5 times every 3 hours.

\section{Abnormal development of PMCs}

P. nigra L. is one of original species in sect. Aigeiros, meiosis of which was conservative under natural condition (Xu et al., 2011). However, colchicine treatment made development of PMCs exhibits various abnormalities almost at all the stages of meiosis (Fig. 4). Fig. 4A-F showed abnormal separation of chromosome and Fig. 4G-L showed abnormal division of cytoplasm.



Fig. 4. Abnormal development of PMCs (bar=10 $\mu \mathrm{m})$ A) PMC with three uneven nuclears; B) Spindle with three poles; C) Perpendicular spindle; D) Chromosome separating uneven; E) PMC with three nuclears; F) Two nuclears with unseparated cytoplasm; G) Dyad; H) Four nuclears with cytoplasm failing to separate; I) Uneven tetrad; J) Uneven tetrad with no cytoplasm in one cavity; $\mathrm{K}, \mathrm{L}$ ) Uneven triad 


\section{Discussion}

Colchicine is known as an alkaloid extracted from Colchicum autumnale L. that influence the organization of the cytoskeletal network via interactions with its tubular structures (Jordan and Wilson, 1999). In A. thaliana, colchicine interfered not only with the biosynthesis of antioxidants such as PQ and UQ (Ernster and Dallner, 1995; Wanke et al., 2000), but also with the enzymatic antioxidant system (Drążkiewicz et al., 2003). These influences potentially cause extensive injuring of orgains and tissues and blocked the subsequent development. Treatment of ungerminated seed in Platanus acerifolia with colchicine produced $40 \%$ tetraploid seedlings but the deleterious effect of colchicine on subsequent root growth made no seedlings grow up (Liu et al., 2007). In loquat (Eriobotrya japonica (Thunb.) Lindl.), survival was significantly lower in all treated plants independently of the treatment duration and colchicine dose (Blasco et al., 2014). In colchicine induction of Populus tomentosa $\times P$. bolleana, pollen production reduced significantly due to the increase of injection times (Kang et al., 1999). Zhang's research in Populus popularis revealed that catkins were blackened and abscised prematurely after injection when concentration of colchicine was raised to $0.8 \%$ (Zhang, 2006).

In our study, chemical damage brought the buds more significant change. Blackened appearance of bud and catkin peak (even whole in some case) (Fig. 2B, C, E, F) probably due to the influence of colchicine with the enzymatic antioxidant system which caused the accumulation of pigment. Sere and blackened catkins did not elongate and abscised prematurely which had similar performance with catkins after injection in Zhang's research (Zhang, 2006). Fig. $2 \mathrm{H}$, I showed smaller size of the whole catkins after injection compared with normal catkins, which leaded to the decline of pollen production directly. Deleterious effect of colchicine may explain the development inhibition of catkins and reduction of pollen production. Damage would become more serious with the increased concentration of colchicine and times of injection. In conclusion, high concentration of colchicine and too many times of injection leaded to the decline of pollen production.

In previous studies, parallel, fused spindles and premature cytokinesis were considered to be leading dyad formation (Zhang and Kang, 2010). However, in our study, parallel spindles (Fig. 4B) were observed both in experimental group and control group suggesting that parallel spindles were not the main reason for dyad formation in $P$. nigra L. Fused spindles and dyad (Fig. 4A and $G$ ) were present only in experimental group, which indicated that dyad formation probably due to fused spindles and premature cytokinesis.

Tripolar spindles (Fig. 4C, D and E) and triad (Fig. 4J and $\mathrm{K}$ ) presented widely in experimental group, which were main, various abnormalities in meiosis of PMCs. Most tripolar spindles and triad were uneven (Fig. 4C, D and J) while equilateral spindles and equipotent triad (Fig. 4E and $\mathrm{K})$ were also observed. In Fig. 4D, two spindles at meiosis II were half fused to form tripolar spindles, which was responsible for one $2 n$ pollen and two $I n$ pollen.
Perpendicular spindle (Fig. 4F) in this study presented as a cross and chromosomes were compline which was distinct from other studies (Zhang and Kang, 2010; Tian et al., 2015). Besides, we observed other various abnormalities such as unseparated cytoplasm (Fig. $4 \mathrm{G}$ and L) and uneven tetrad (Fig. 4I).

It was noticed that PMCs which had abnormal spindles in meiosis I were hardly observed because the chromosome failing to separate in meiosis I would complete meiosis II which seemed as a normal meiosis I. In this case, cytoplasm divided at telophase II to form a dyad and turned into two FDR-type $2 n$ pollen, therefore the theoretical rate of $2 n$ pollen was $100 \%$. Half-fused spindles at meiosis II mentioned above could produce FDR or SDR-type $2 n$ pollen and theoretical rate of $2 n$ pollen in this condition was $33.33 \%$. Fused spindle at meiosis II could also cause $2 n$ pollen while types of which were not confirmed. In this condition, theoretical rates of $2 n$ pollen were $100 \%$. In this study, high rate of $2 n$ pollen in some treatment combination probably due to FDR or/and fused spindle at meiosis II.

This study observed development abnormalities of PMCs after colchicine treatment and revealed the cytological mechanism of $2 n$ pollen formation in Populus nigra L. for the first time, which act as guidance for the following induction of $2 n$ pollen in sect. Aigeiros. The best treated combination of colchicine treatment was obtain and the highest rate of $2 n$ pollen was reached at $87.11 \%$ (even $100 \% 2 n$ pollen in some floral buds) which made the utilization of $2 n$ pollen to polyploid hybridization in sect. Aigeiros possible.

\section{Acknowledgements}

This research was supported by Medium and Long Scientific Research Project for Young Teachers in Beijing Forestry University(2015ZCQ-SW-02), the Project of National Natural Science Foundation of China (No. 31370658), "948” Project of China (2014-4-59), Program for Changjiang Scholars and Innovative Research Team in University (IRT13047).

\section{Conflict of Interest}

The authors declare that there are no conflicts of interest related to this article.

\section{References}

Bastiaanssen H, Berg P, Lindhout P, Jacobsen E, Ramanna MS (2010). Postmeiotic restitution in $2 n$-egg formation of diploid potato. Heredity 81(1):20-27.

Blasco M, Badenes M, Naval M (2014). Colchicine-induced polyploidy in loquat (Eriobotrya japonica (Thunb.) Lindl.). Plant Cell, Tissue and Organ Culture 120(2):453-461.

BretagnolleF, Thompson JD (2010). Tansley review no 78 - gametes with the somatic chromosome number: mechanisms of their formation and role in the evolution of autopolyploid plants. New Phytologist 129(1):1-22. 
946

Dewitte A, Eeckhaut T, Huylenbroeck JV, Bockstaele EV (2010). Meiotic aberrations during $2 n$ pollen formation in Begonia. Heredity $104(2): 215-223$.

Dewitte A, Laere KV, HuylenbroeckJV (2012). Use of $2 n$ gametes in plant breeding. In: Abdurakhmonov IY (Ed). Plant breeding. InTech Open Access Publisher, Croatia pp 59-86.

Drazkiewicz M, Skórzyńska-Polit E, Wanke M, Swiezewska E (2003). The activity of antioxidant enzymes in Arabidopsis thaliana exposed to colchicine and $\mathrm{H}_{2} \mathrm{O}_{2}$. Cellular \& Molecular Biology Letters 8:777-781.

Ernster L, Dallner G (1995). Biochemical, physiological and medical aspects of ubiquinone function. Biochimica et Biophysica Acta 1271(1):195204.

Jongedijk E, Ramanna MS, Sawor Z, Hermsen JGT (1991). Formation of first division restitution (FDR) $2 n$-megaspores through pseudohomotypic division in $d s-1$ (desynapsis) mutants of diploid potato: routine production of tetraploid progeny from $2 \times \mathrm{FDR} \times$ 2×FDR crosses. Theoretical \& Applied Genetics 82(5):645-656.

Jordan MA, Wilson L (1999). The use and action of drugs in analyzing mitosis. Methods in Cell Biology 61:267-295.

Kang XY (2002). Mechanism of $2 n$ pollen occurring in Chinese white poplars. Journal of BeijingForestryUniversity 24(5-6):67-70.

Kang XY, Zhu ZD (1997). A study on the $2 n$ pollen vitality and germinant characteristics of white poplars. Acta Botanica Yunnanica 19(4):395401.

Kang XY, Zhu ZD, Lin HB (1999). Study on the effective treating period for pollen chromosome doubling of Populus tomentosa $\times$ P. bolleana. ScientiaSilvae Sinicae 35:21-24.

Kang XY, Zhu ZD, Zhang ZY (2000). Suitable period of high temperature treatment for $2 n$ pollen of Populus tomentosa $\times P$. bolleana. Journal of BeijingForestryUniversity 22(3):1-4.

Li Y (2001). Advances in studies of triploid poplar breeding. Bulletin of Botany 18:451-458.

Li Y, Zhu Z, Tian Y, Zhang Z, Kang X (2000). Obtaining triploids by high and low temperature treating female flower buds of white poplar. Journal of BeijingForestry University 22(5):7-12.

Lim KB, Ramanna MS, Jong J, Jacobsen E, Tuyl J (2001). Indeterminate meiotic restitution (IMR): a novel type of meiotic nuclear restitution mechanism detected in interspecific lily hybrids by GISH. Theoretical \& AppliedGenetics 103(2-3):219-230.

Liu G, Li Z, Bao M (2007). Colchicine-induced chromosome doubling in Platanus acerifolia and its effect on plant morphology. Euphytica 157(12):145-154.

Mashkina OS, Burdaeva LM, Belozerova MM (1989). Method of obtainingdiploid pollen of woody species. Lesovedenie 1:19-25.
Otto SP, Whitton J (2000). Polyploid incidence and evolution. Annual Review ofGenetics 34(1):401-437.

Ramanna MS (1983). First division restitution gametes through fertile desynaptic mutants of potato. Euphytica 32(2):337-350.

Ramanna MS, Agj K, Jacobsen E (2003). Occurrence of numerically unreduced $(2 n)$ gametes in Alstroemeria interspecific hybrids and their significance for sexual polyploidisation. Euphytica 133(1):95-106.

Salmon ED, Mckeel M, Hays T (1984). Rapid rate of tubulin dissociation from microtubules in the mitotic spindle in vivo measured by blocking polymerization with colchicine. Journal of Cell Biology 99(3):10661075.

Taschetto OM, Pagiarini MS (2003). Occurrence of $2 n$ and jumbo pollen in the Brazilian ginseng (Pfaffia glomerata and P. tuberosa). Euphytica 133(1):139-145.

Tian J, WangJ, Dong L, Dai F, WangJ (2015). Pollen variation as a response to hybridisation in Populus L. section Aigeiros Duby. Euphytica 206(2):1-11.

Wanke M, Dallner G, Swiezewska E (2000). Subcellular localization of plastoquinone and ubiquinone synthesis in spinach cells. BBABiomembranes 1463(1):188-194.

Xi XJ, Jiang XB, Li D, Guo LQ, Zhang JF, Wei ZZ, Li BL (2011). Induction of $2 n$ pollen by colchicine in Populus $\times$ popularis and its triploids breeding. Silvae Genetica 60(1-6):155-160.

Xu WT, Zhao J, Zhao N, Guo LQ, Zhang JG, Xia C, Zhang JF (2011). Meiosis of pollen mother cells and their asynchrony in Populus nigra $\mathrm{L}$. Acta Botanica Boreali-Occidentalia31(10):1981-1987.

Zhang JF (2006). Mechanisms of $2 n$ pollen formation and its inducing technique of poplar in section Aigeiros. Beijing: Beijing Forestry University.

Zhang S, Qi L, Chen C, Li X, Song W, Chen R, Han S (2004). A report of triploid Populus of the section Aigeiros. Silvae Genetica 53(1-6):69-75.

Zhang ZY, Kang XY (2010). Cytological characteristics of numerically unreduced pollen production in Populus tomentosa Carr. Euphytica 173(2):151-159.

Zhou S, Ramanna M, Visser R, Tuyl J (2008). Analysis of the meiosis in the $\mathrm{F}_{1}$ hybrids of Longiflorum $\times$ Asiatic (LA) of lilies (Lilium) using genomic in situ hybridization. Journal of Genetics and Genomics 35(11):687-695.

Zhu ZD, Kang XY, Zhang ZY (1998). Studies on selection of natural triploids of Populus tomentosa. Scientia Silvae Sinicae 34(4):22-31. 